\title{
Encounters in the ONC - observing imprints of star-disc interactions
}

\author{
C. Olczak ${ }^{1}$, S. Pfalzner ${ }^{1}$, and A. Eckart ${ }^{1,2}$ \\ 1 I. Physikalisches Institut, Universität zu Köln, Zülpicher Str.77, 50937 Köln, Germany \\ e-mail: olczak@ph1.uni-koeln.de \\ 2 Max-Planck-Institut für Radioastronomie, Auf dem Hügel 69, 53121 Bonn, Germany
}

Received 18 March 2008 / Accepted 3 June 2008

\begin{abstract}
Context. The external destruction of protoplanetary discs in a clustered environment acts mainly due to two mechanisms: gravitational drag by stellar encounters and evaporation by strong stellar winds and radiation. It is a fundamental question whether either of these mechanisms is important for the stellar evolution and planet formation process in young star clusters.

Aims. We focus on the effect of stellar encounters in young dense clusters and investigate whether there are any observables that could trace this mechanism and its impact on disc evolution. If encounters play a role in disc destruction, one would expect that stars devoid of disc material would show unexpectedly high velocities as an outcome of close interactions. We want to quantify this effect by numerical simulations and compare it to observations.

Methods. As a model cluster we chose the Orion Nebula Cluster (ONC). We reanalyzed observational data of the ONC to find encounter signatures in the velocity distribution and a possible correlation with signatures of circumstellar discs. We use the nbody6++ code to model the dynamics of an ONC-like cluster and analyze the velocity distribution and the disc-mass loss due to encounters. Results. We found from the observational data that 8 to 18 stars leave the ONC with velocities several times the velocity dispersion. The majority of these high-velocity stars are young low-mass stars $\left(t \lesssim 10^{5} \mathrm{yr}, m \approx 0.2-0.3 M_{\odot}\right)$, among them several lacking infrared excess emission. Interestingly, the high-velocity stars are found only in two separate regions of the ONC $-i)$ close to the cluster centre and ii) in the outer cluster region. Our simulations give an explanation for the location of the high-velocity stars and provide evidence for a strong correlation between location and disc destruction.

Conclusions. The high-velocity stars can be explained as the outcome of close three-body encounters; the partial lack of disc signatures is attributed to gravitational interaction. The spatial distribution of the high-velocity stars reflects the initial structure and dynamics of the ONC. Our approach can be generalized to study the evolution of other young dense star clusters, like the Arches cluster, back in time.
\end{abstract}

Key words. stellar dynamics - methods: N-body simulations - stars: planetary systems: protoplanetary disks - methods: data analysis - stars: kinematics

\section{Introduction}

According to current knowledge, planetary systems form from the accretion discs around young stars. These young stars are in most cases not isolated but are part of a cluster (e.g. Lada \& Lada 2003). Densities in these cluster environments vary considerably, spanning a range of $10 \mathrm{pc}^{-3}$ (e.g. $\eta$ Chameleontis) to $10^{6} \mathrm{pc}^{-3}$ (e.g. Arches Cluster). It is still an open question as to how far interactions with the surrounding stars influence planet formation in dense young clusters $\left(n \gtrsim 10^{4} \mathrm{pc}^{-3}\right)$. These discs disperse over time (Haisch et al. 2001; Hillenbrand 2002; SiciliaAguilar et al. 2006; Currie et al. 2008) and in dense clusters the disc frequency seems to be lower in the core (e.g. Balog et al. 2007). This is attributed to external violent processes like photoevaporation or encounter-induced disc mass loss, mainly caused by the massive stars that are concentrated in the cluster core.

Earlier numerical investigations seemed to indicate that photoevaporation should by far dominate the external disc destruction (Scally \& Clarke 2001; Adams et al. 2004). The question of whether encounters play a vital role in the formation process of stars and planets has been studied far less and is still open (e.g. Adams et al. 2006). Only recently has it been shown that stellar encounters do have an effect on the discs surrounding stars in a young dense cluster (Olczak et al. 2006;
Pfalzner et al. 2006; Pfalzner 2006; Moeckel \& Bally 2006, 2007a,b; Pfalzner \& Olczak 2007a,b; see also the review by Zinnecker \& Yorke 2007). The importance of encounters has been underestimated because in previous studies the focus has been on encounters between solar mass stars (e.g. Clarke \& Pringle 1991; Heller 1995). However, discs are most affected when the masses of the stars involved in the encounter are unequal (Olczak et al. 2006; Moeckel \& Bally 2007b). Moreover, the massive stars in the center of a stellar cluster act as gravitational foci for the lower mass stars and are thus subject to repeated encounters. These accumulated perturbations can lead to a total destruction of a massive star's disc (Pfalzner et al. 2006).

The main focus of this paper is the question of whether there is direct observational evidence for encounters among star-disc systems in young clusters and for disc-mass loss due to encounters. It is difficult to distinguish observationally whether photoevaporation or gravitational interaction are responsible for the loss of (outer) discs. The reason is that in both cases interaction with a massive star is the most destructive process. Thus the observation of a decreased disc frequency in the cluster core, as mentioned above, does not allow one to favor either of the two mechanisms. The difficulty of tracing stellar encounters directly by observations is their short duration. Direct imaging of tidal 
tails of a disc would be the best proof but the probability of such an event is very low: the perturbed disc circularizes and tidal tails dissipate on a time scale of $\lesssim 1000 \mathrm{yr}$. Nevertheless, there exist observations of tidal tails or spiral arms in star-disc systems (e.g. Beust et al. 2005; Cabrit et al. 2006; Lin et al. 2006), but often it is unclear whether these can be attributed to the passage of an unbound perturber or a binary companion or gravitational instabilities caused by a giant planet. Due to the short dissipation time scale of encounter-induced tidal tails, there would be a high probability for the encounter partner still to be located close $\left(\lesssim 10^{4} \mathrm{AU}\right)$ to the perturbed disc. However, source confusion in dense young clusters like the ONC, where stellar encounters are frequent enough to study the effect on stellar discs, would prevent a clear identification. Set in relation to the rate of encounters in which prominent tidal tails may be formed, we estimate an upper limit of just four candidate stars for a direct observation of tidal tails in a protoplanetary disc due to encounters in the ONC (see Appendix A for a detailed calculation). However, an unambiguous imprint of an encounter among stars is the high velocity of a star which has been expelled in a close gravitational interaction, mostly as a result of a three-body encounter (see Heggie 1975). Thus an analysis of the velocity distribution of a cluster is the key to finding candidates of close encounters between young stars.

Here the $\mathrm{ONC}$ is used as a prototype young cluster because it is one of the best-studied regions in our galaxy, and the only young dense cluster for which velocities of more than 1000 of its members have been determined (Jones \& Walker 1988). In addition, its high density suggests that stellar encounters might be relevant for the evolution of circumstellar discs. Throughout this work we assume that initially all stars are surrounded by protoplanetary discs. This is justified by observations that reveal disc fractions of nearly $100 \%$ in very young star clusters (e.g. Haisch et al. 2000; Lada et al. 2000; Haisch et al. 2001; Hillenbrand 2005).

In Sect. 2 we present results from a search for candidate stars of close encounters in the publicly available observational data of the ONC. For this purpose we have reanalyzed the investigations of Jones \& Walker (1988), Hillenbrand (1997), and Hillenbrand et al. (1998) for stellar velocities and infrared excess. The basic properties of the ONC used for our numerical model are described in Sect. 3. There we also discuss theoretical estimates and observational data related to binary populations and encounters. Afterwards we present results from a numerical approach to this problem (Sect. 4). Observational and numerical results are summerized and discussed in Sect. 5.

\section{Observational data}

Our search for candidate stars of close encounters from observational data concentrates on velocity surveys of Orion. There exists only one large data set of the ONC that provides velocity information for several hundred stars, the proper motion survey of Jones \& Walker (1988). Fortunately, the same stars have been analyzed by Hillenbrand et al. (1998) for infrared excess emission as an indicator of circumstellar material. They defined a quantity measuring the magnitude of the near-infrared excess, $\Delta\left(I_{\mathrm{C}}-K\right)$,

$\Delta\left(I_{\mathrm{C}}-K\right)=\left(I_{\mathrm{C}}-K\right)_{\text {observed }}-0.5 A_{V}-\left(I_{\mathrm{C}}-K\right)_{\text {photosphere }}$,

where the first term is the observed $I_{\mathrm{C}}-K$ color, the second term the contribution of reddening calculated from the extinction values derived from $V-I_{C}$ colors as in Hillenbrand (1997), and the third term the contribution of the underlying stellar photosphere. This measure of the near-infrared excess, $\Delta\left(I_{C}-K\right)$, traces only the innermost $(<0.1 \mathrm{AU})$ part of the circumstellar disc. Hence, the absence of such emission is not to be confused with a complete absence of a protoplanetary disc, yet is dependent on a number of parameters, such as disc accretion rate, inclination, inner hole, and stellar mass and radius (Hillenbrand et al. 1998). However, in the following, we will refer to stars that lack infrared excess emission as "discless", emphasizing that these stars may be well surrounded by circumstellar material but do not show the typical infrared excess of a young star-disc system.

In addition Hillenbrand (1997) has investigated stellar properties like mass and age. The investigation is based on optical photometric and spectroscopic data and covers only about half of the stellar population of the ONC, while the more embedded stars are not accessible at this wavelength. However, Hillenbrand (1997) states that the investigated stellar sample is representative of the entire population of the ONC. Masses and ages were derived from an HR diagram via comparison with theoretical pre-main sequence evolutionary tracks. This method leaves some uncertainty as to the absolute stellar age ( $\sim 0.5 \mathrm{dex}$, see Appendix B) and mass calibrations, with mass deviations of about factors of two and age differences of several Myr among different models. Additionally, photometric errors translate into uncertainties of the derived masses, but this is significant only for stars more massive than $M \approx 1.5 M_{\odot}$. However, all these sources of uncertainties are less relevant for the present investigation because (i) the stars of interest are of low mass as will be shown later; and (ii) the derived conclusions are based mainly on relative masses and ages.

We have merged the data of the three investigations described and excluded stars for which measurements of proper motion or infrared excess are missing, resulting in a database of 655 stars. In order to achieve the most secure distinction possible between stars with infrared excess and pure photospheric emission, we adopted the criterion of Sicilia-Aguilar et al. (2005) and excluded all stars that have an infrared excess $\Delta\left(I_{\mathrm{C}}-K\right)$ in the range $0<\Delta\left(I_{\mathrm{C}}-K\right)<0.5$. A star is classified as discless if $\Delta\left(I_{\mathrm{C}}-K\right) \leq 0$, or as a star-disc system if $\Delta\left(I_{\mathrm{C}}-K\right) \geq 0.5$. This additional selection criterion reduces the sample of stars that have been used for this investigation to 405, among them 266 star-disc systems and 139 stars lacking any excess emission.

In Fig. 1 we show the velocity distribution of stars with and without discs, adopting a distance of $420 \mathrm{pc}$ to the ONC (cf. Jeffries 2007; Menten et al. 2007). The velocity distribution has been built by binning each component of the proper motion, $\mu_{x}$ and $\mu_{y}$, of each star separately, not its total two-dimensional motion, or in other words by summing the Gaussian velocity distribution of each spacial direction, $x$ and $y$, which again results in a Gaussian. The reason not to bin the total two-dimensional motion is the improvement of statistics due to the twice as large data set. For comparison, a Gaussian with a one-dimensional velocity dispersion $\sigma=\sigma_{1 \mathrm{D}}^{\mathrm{JW}}=2.5 \mathrm{~km} \mathrm{~s}^{-1}$, as derived by Jones \& Walker (1988), is superimposed (dashed line). The distribution shows the expected features: At velocities $\mu_{x, y} \lesssim 3 \sigma_{1 \mathrm{D}}^{\mathrm{JW}}$ the shape is approximately Gaussian, though a distinct peak at zero velocity is present. This peak is only prominent for the $x$-components of the proper motion but is independent of the applied binning. We suppose that this is an artifact of the plate reduction technique used by Jones \& Walker (1988). At higher velocities, $\mu_{x, y} \gtrsim 3 \sigma_{1 \mathrm{D}}^{\mathrm{JW}}$, there is an overabundance of stars when compared with the theoretically expected Gaussian distribution. In this regime stars are not bound energetically to the cluster (see 

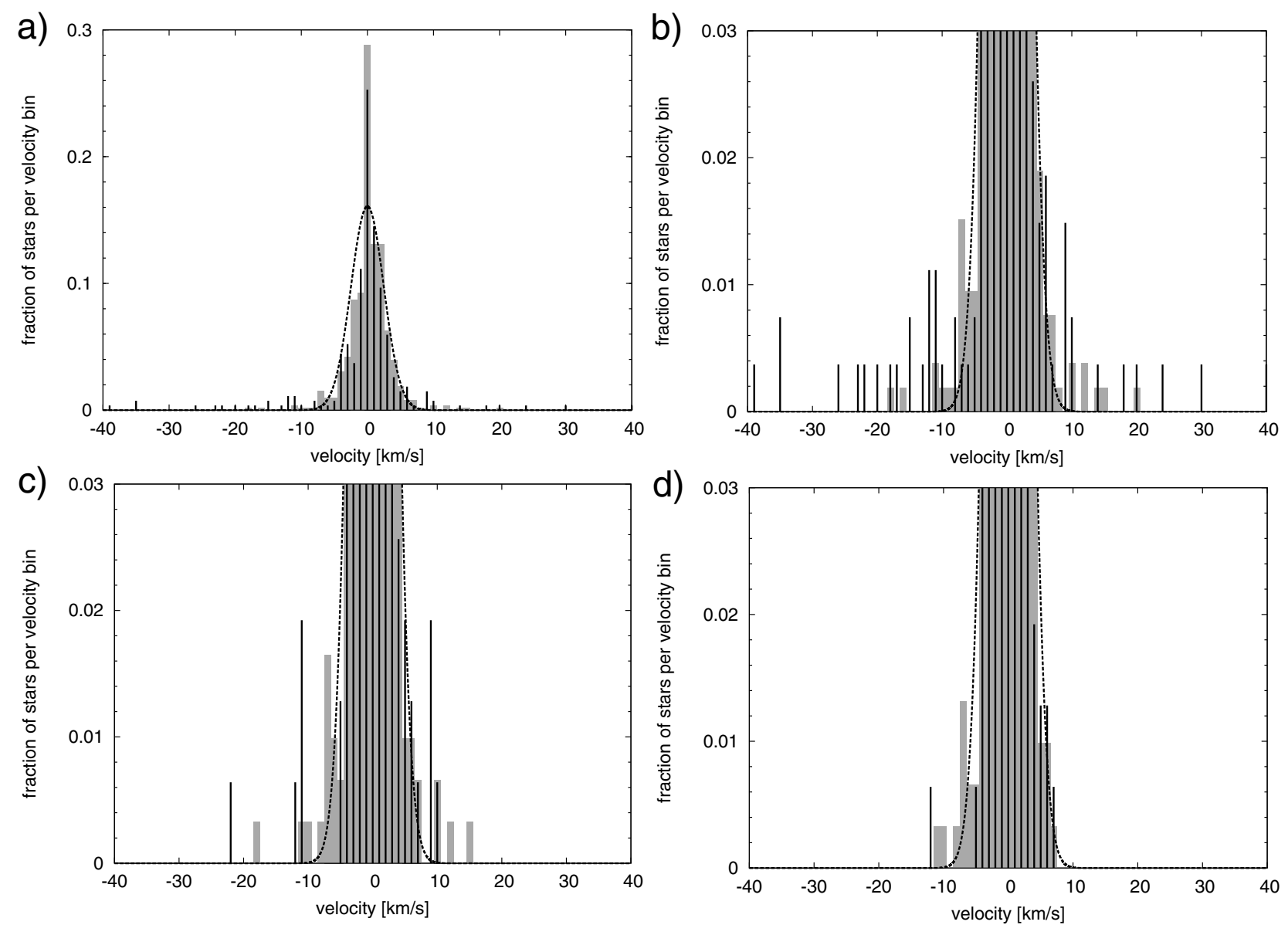

Fig. 1. Velocity distribution from observational data, adopting a distance of $420 \mathrm{pc}$ to the ONC (cf. Jeffries 2007; Menten et al. 2007). See text for details of its construction. The bin width is $0.7 \mathrm{~km} \mathrm{~s}^{-1}$. The stars have been divided into two groups according to the disc signature $\Delta\left(I_{\mathrm{C}}-K\right.$ ) (see text): grey boxes represent star-disc systems, lines represent discless stars. For comparison a Gaussian with dispersion $\sigma=\sigma_{1 \mathrm{D}}^{\mathrm{JW}}=2.5 \mathrm{~km} \mathrm{~s}{ }^{-1}\left(\mathrm{Jones}^{\mathrm{J}}\right.$ \& Walker 1988) is superposed (dashed line). a) Complete sample of stars in the ONC with available proper motions from Jones \& Walker (1988) and infrared excess from Hillenbrand et al. (1998). b) Like a), but zoomed into the lower part. c) Like b), but with restricted stellar ages (see text). d) Like b), but with restricted stellar ages and velocity errors as described in the text.

Binney \& Tremaine 1987, Eq. (8-3)). In the following we will refer to these stars as "high-velocity stars".

The number of high-velocity stars, $N_{\text {hvs, obs }}=50$, is remarkably high, much higher than the theoretically expected $N_{\text {hvs }} \lesssim 15$, as derived in Sect. 3.2. In fact, $N_{\mathrm{hvs}}$ obs should be even lower than the theoretical $N_{\text {hvs }}$ because only about $1 / 5$ of the ONC stellar population is covered by the observational data. The reason is most likely a contribution from foreground stars, which show large proper motions due to projection effects. In order to exclude probable foreground stars from the sample of ONC stars, we plot in Fig. 2 age vs. velocity for the high-velocity stars, again for the two groups of discless stars and star-disc systems. Two opposite trends are visible: The discless stars with ages $t>1 \mathrm{Myr}$ have a $\sim 10$ times higher maximum velocity than younger ones $\left(\sim 200 \mathrm{~km} \mathrm{~s}^{-1}\right.$ compared to $\left.\sim 20 \mathrm{~km} \mathrm{~s}^{-1}\right)$ and a twice as high mean velocity $\left(\sim 13 \mathrm{~km} \mathrm{~s}^{-1}\right.$ compared to $\left.\sim 30 \mathrm{~km} \mathrm{~s}^{-1}\right)$. This strong correlation is likely due to a large fraction of foreground objects among the stars with ages $t>1 \mathrm{Myr}$, and since this age boundary is consistent with the mean cluster age, it marks a conservative upper limit to the age of probable cluster members. Conversely, the star-disc systems are similarly distributed in both age groups, with comparable maximum and median velocities $\left(\sim 25 \mathrm{~km} \mathrm{~s}^{-1}\right.$ and $\sim 11 \mathrm{~km} \mathrm{~s}^{-1}$, respectively). However, we exclude all stars with ages $t>1 \mathrm{Myr}$ in the further discussion for three reasons: (i) the age of these stars exceeds our simulated cluster age, so they are dynamically distinct; (ii) at least some of these stars might not be members of the ONC due to their high age (and velocity); and (iii) protoplanetary discs with ages $t>1$ Myr may be subject to significant evolution due to internal processes ${ }^{1}$, so that the effect of encounters could not be quantified.

Of the group of high-velocity stars younger than $1 \mathrm{Myr}$, the "probable high-velocity cluster members", about half show disc signatures, the rest does not. The fact that stars with velocities of several tens of $\mathrm{km} \mathrm{s}^{-1}$ are not present in this group is in accordance with dynamical estimates: the probability of both the generation and detection of stars with such high velocities is very low due to the need for a very close approach and short traverse of the cluster.

The uncertainties in the estimated ages of stars with and without infrared excess emission do not alter the fact that some of the youngest high-velocity stars lack infrared excess. This is noteworthy because in the early stages of stellar evolution one would expect accretion rates to be high and circumstellar

1 According to studies of properties and evolution of protoplanetary discs in young clusters (e.g. Hillenbrand 2005; Sicilia-Aguilar et al. 2006), it is valid to assume that the protoplanetary discs in the ONC have been only marginally subject to internal disc processes. As such, we assume in the following that the effect of external processes on discs, i.e. photevaporation and encounters, have not been masked by internal processes at the current age of the ONC. 


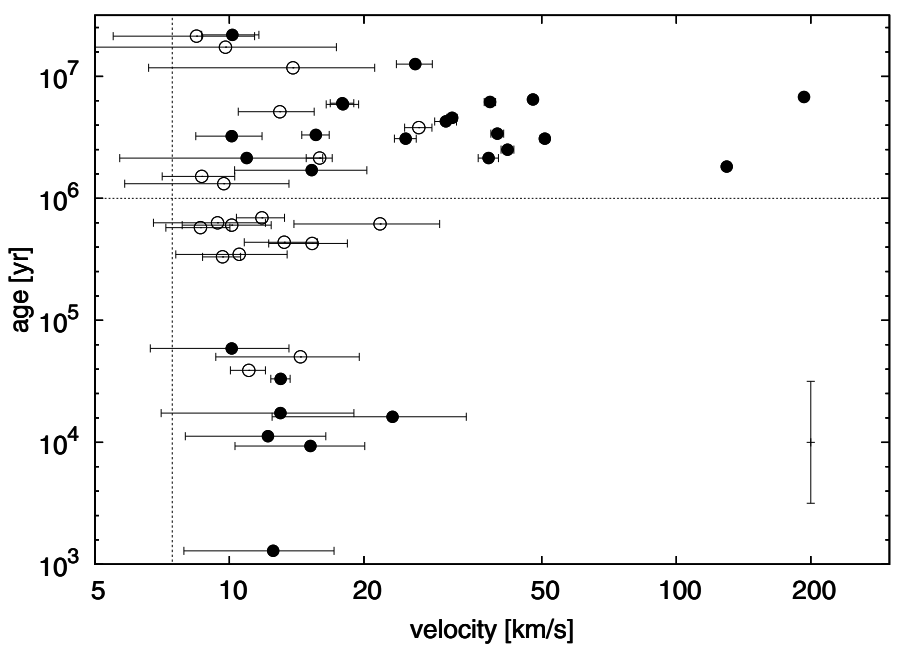

Fig. 2. Distribution of age and velocity of the high-velocity stars in the ONC, classified as star-disc systems (open circles) and discless stars (filled circles). The mean age of the ONC, $t=1 \mathrm{Myr}$, and the minimum one-dimensional velocity of the high-velocity sample, $3 \sigma_{1 \mathrm{D}}^{\mathrm{JW}}$, are indicated by the horizontal and vertical dashed line, respectively. The uncertainty of the determined ages of $\sim 0.5$ dex (see Appendix B for the derivation) is indicated by the vertical error bar. At high velocities the (horizontal) velocity error bars are smaller than the symbol size.

material to be close enough to the star that significant excess emission can be detected. However, because the excess emission measured by $\Delta\left(I_{\mathrm{C}}-K\right)$ originates close to the stellar surface, it is strongly dependent on the geometry and orientation of the disc. A more robust and sensitive indicator of circumstellar discs is the $K-L$ color (Meyer et al. 1997; Haisch et al. 2000), tracing material out to radii of $\sim 0.1 \mathrm{AU}$ (Haisch et al. 2005). We have thus cross-checked the "discless" stars with $\Delta\left(I_{\mathrm{C}}-K\right) \leq 0$ for excess emission at longer wavelengths. The results are shown in Table 1.

Two of the stars previously determined as "discless" do show a typical emission signature of warm circumstellar matter. Four high-velocity stars remain that lack infrared excess. We cannot determine whether the pure photospheric colors point to the absence of a circumstellar disc. We will discuss this possibility later.

We have plotted the positions and velocity vectors of the selected probable high-velocity cluster members in Fig. 3a. Two features are apparent: (i) Most high-velocity stars are concentrated in the inner tenths of a parsec around the most massive ONC member, $\theta^{1} \mathrm{C}$ Ori; and (ii) "outliers" are located more than $1 \mathrm{pc}$ from the cluster center with some stars moving in radial directions away from the cluster centre. Although one would expect the former, as most encounters usually happen in the dense cluster centre, there are two unexpected features: (i) The region between the cluster centre and outer cluster parts, $0.4 \mathrm{pc} \lesssim r \lesssim 1.0 \mathrm{pc}$, is devoid of high-velocity stars; and (ii) the velocity vectors of several stars in the outer cluster parts do not point away from the cluster centre (as if these stars had an encounter in the less dense cluster parts). In order to be confident that the encounter candidate stars are indeed high-velocity stars and not affected by observational uncertainties, we restrict our sample of high-velocity stars to maximum velocity errors of less than $30 \%$ or below $2.0 \mathrm{~km} \mathrm{~s}^{-1}$. This reduces the number of highvelocity stars to a total of only eight, among them just one star without infrared excess, JW 45. The corresponding positionvelocity diagram is shown in Fig. 3 b (see also the corresponding velocity distribution in Fig. 1d). However, apart from the
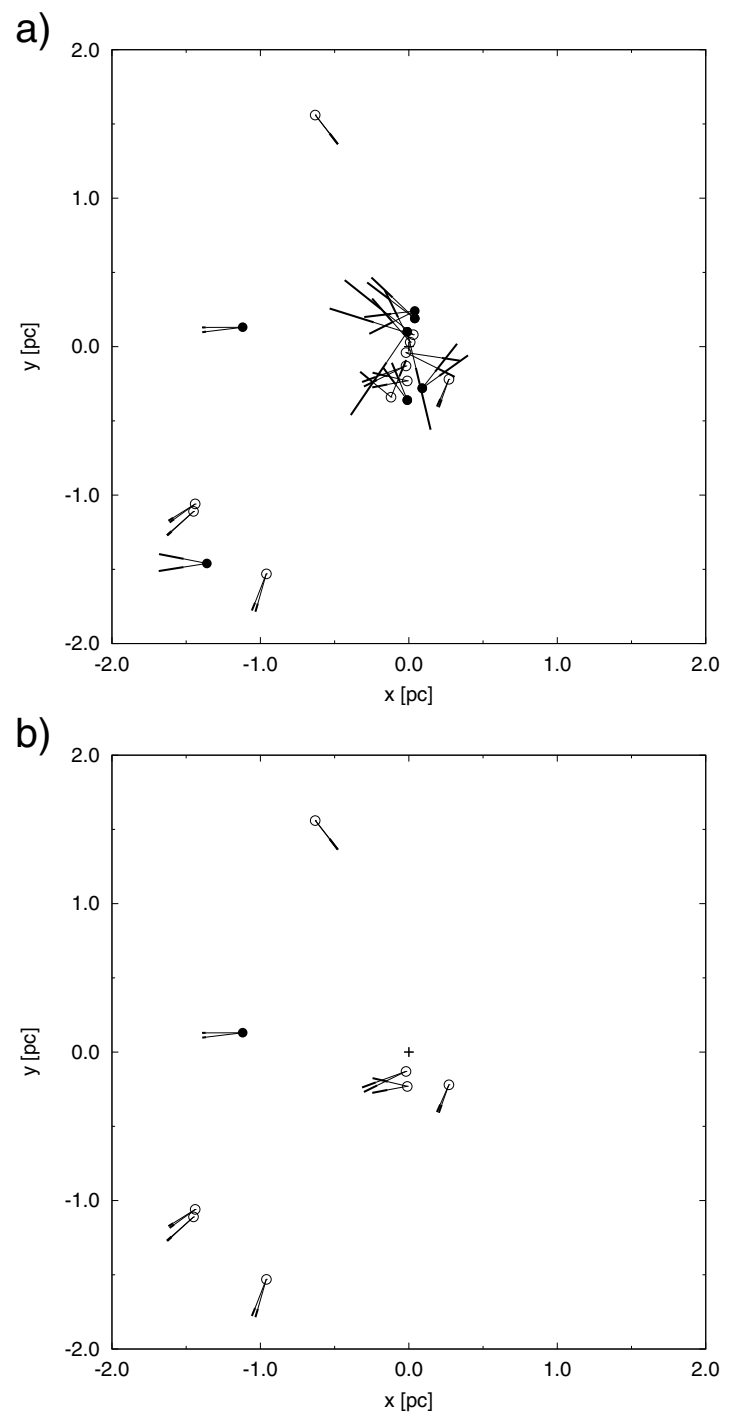

Fig. 3. Positions and velocities of high-velocity star-disc systems (open circles) and discless stars (filled circles) in the ONC. The frames are centered on the most massive star, $\theta^{1} \mathrm{C}$ Ori, which is marked by a cross. Thin lines indicate the distance (in pc) a star would have moved in a period of $2 \times 10^{4} \mathrm{yr}$. Thick lines and the opening angle reflect the error of the magnitude and the direction of the proper motion, respectively. a): sample of all high-velocity stars with ages $<1 \mathrm{Myr}$. b): restricted sample of high-velocity stars with ages $<1$ Myr: only stars fulfilling the constraints on the velocity errors as dicussed in the text are shown.

concentration of high-velocity stars close to the cluster centre, the same features as in Fig. 3a are apparent. The reason for the different central concentration is the strong acceleration of stars close to the cluster centre which results in large errors of the derived proper motions. Consequently our velocity error criterion preferentially excludes stars close to the cluster centre. We will address the particular features in Sect. 4, where we present the results from numerical simulations of our dynamical model of the ONC.

Below we review the known properties of the ONC, which we use to model the cluster in an as realistic a way as possible.

\section{Structure and dynamics of the ONC}

The ONC is a rich stellar cluster with about 4000 members with masses $m \geq 0.08 M_{\odot}$ in a volume $\sim 5 \mathrm{pc}$ across 
Table 1. Properties of discless high-velocity stars in the ONC.

\begin{tabular}{|c|c|c|c|c|c|c|c|c|c|c|c|c|c|c|}
\hline $\begin{array}{l}\mathrm{ID}^{a} \\
{[\mathrm{JW}]}\end{array}$ & $\begin{array}{r}r^{b} \\
{[\mathrm{pc}]}\end{array}$ & $\begin{array}{r}\mu_{x}{ }^{c} \\
{\left[\mathrm{~km} \mathrm{~s}^{-1}\right]}\end{array}$ & $\begin{array}{r}\sigma_{x} \\
{\left[\mathrm{~km} \mathrm{~s}^{-1}\right]}\end{array}$ & $\begin{array}{r}\mu_{y} \\
{\left[\mathrm{~km} \mathrm{~s}^{-1}\right]}\end{array}$ & $\begin{array}{r}\sigma_{x} \\
{\left[\mathrm{~km} \mathrm{~s}^{-1}\right]}\end{array}$ & $\begin{array}{r}P^{d} \\
{[\%]}\end{array}$ & $\begin{array}{r}J^{e} \\
{[\mathrm{mag}]}\end{array}$ & $\begin{array}{r}H \\
\text { [mag] }\end{array}$ & $\begin{array}{r}K \\
{[\mathrm{mag}]}\end{array}$ & $\begin{array}{r}L \\
{[\mathrm{mag}]}\end{array}$ & $\mathrm{SpT}^{f}$ & $\begin{array}{r}m^{g} \\
{\left[M_{\odot}\right]}\end{array}$ & $\begin{array}{r}\log t^{h} \\
{[\mathrm{yr}]}\end{array}$ & excess \\
\hline JW 19 & 1.99 & $\frac{1}{-12.2}$ & $\frac{1}{4.2}$ & $\begin{array}{l}\perp \\
\end{array}$ & 2.2 & 84 & 12.98 & 12.26 & 11.87 & & M5.5 & 0.16 & 4.05 & $\overline{10}$ \\
\hline JW 45 & 1.12 & -13.0 & 0.6 & -0.8 & 0.8 & 0 & 8.83 & 8.28 & 8.05 & 7.89 & K3 & 0.94 & 4.52 & no \\
\hline JW 505 & 0.10 & -4.2 & 11.4 & -22.8 & 8.8 & 93 & 12.33 & 11.65 & 11.18 & 10.65 & M2 & 0.20 & 4.21 & $?$ \\
\hline JW 510 & 0.36 & -5.0 & 2.8 & 8.8 & 2.4 & 95 & 13.01 & 12.18 & 11.76 & 11.16 & M5 & 0.14 & 4.77 & yes \\
\hline JW 559 & 0.19 & -11.6 & 3.2 & 9.8 & 3.8 & 99 & 12.43 & 11.74 & 11.30 & 11.04 & M5.5e & 0.16 & 3.97 & no \\
\hline JW 569 & 0.24 & -12.0 & 3.8 & -3.6 & 3.4 & 92 & 12.47 & 11.58 & 11.00 & 10.14 & M3.5 & 0.09 & 3.11 & yes \\
\hline JW 616 & 0.30 & 9.4 & 5.6 & 9.0 & 2.8 & 95 & 13.21 & 12.36 & 11.91 & 11.50 & M3.5 & 0.15 & 4.24 & no \\
\hline
\end{tabular}

${ }^{a}$ Stellar ID from Jones \& Walker (1988). ${ }^{b}$ Projected distance from $\theta^{1} \mathrm{C}$ Ori. ${ }^{c}$ Proper motion and associated error. ${ }^{d}$ Probability of cluster membership. ${ }^{e}$ Apparent magnitude in specified band filter. ${ }^{f}$ Spectral type. ${ }^{g}$ Stellar mass. ${ }^{h}$ Stellar age. ${ }^{i}$ Detection of excess emission.

(Hillenbrand \& Hartmann 1998; Hillenbrand \& Carpenter 2000). Most of the objects are T Tauri stars. The mean stellar mass is about $\bar{m} \approx 0.5 M_{\odot}$ and the half-mass radius $R_{\mathrm{hm}} \approx 1 \mathrm{pc}$ (Scally et al. 2005). Recent studies of the stellar mass distribution (Hillenbrand \& Carpenter 2000; Luhman et al. 2000; Muench et al. 2002; Slesnick et al. 2004) reveal no significant deviation from the generalized IMF of Kroupa (2002),

$\xi(m)= \begin{cases}m^{-1.3}, & 0.08 \leq m / M_{\odot}<0.50 \\ m^{-2.3}, & 0.50 \leq m / M_{\odot}<1.00 \\ m^{-2.3}, & 1.00 \leq m / M_{\odot}<\infty .\end{cases}$

The shape of the system is not perfectly spherical but elongated in the north-south direction. The probable reason for this asymmetry is the gravitational potential of a massive molecular ridge in the background of the cluster, OMC 1 (Hillenbrand \& Hartmann 1998). The mean age of the whole cluster has been estimated to be $t_{\mathrm{ONC}} \approx 1 \mathrm{Myr}$, although a significant age spread of the individual stars is evident (Hillenbrand 1997; Palla \& Stahler 2000). Radio observations by Wilson et al. (1997) show that only a few solar masses of ionized gas are present in the inner $\sim 1 \mathrm{pc}$.

The density and velocity distribution of the ONC resembles an isothermal sphere. The central number density $\rho_{\text {core }}$ in the inner $0.053 \mathrm{pc}$ reaches $4.7 \times 10^{4} \mathrm{pc}^{-3}$ (McCaughrean et al. $2002)$ and makes the ONC the densest nearby $(<1 \mathrm{kpc})$ young stellar cluster. The dense inner part of the ONC, also known as the Trapezium Cluster (TC), is characterized by $R_{\mathrm{TC}} \lesssim 0.3 \mathrm{pc}$ and $N_{\mathrm{TC}} \approx 750$, or $n_{\mathrm{TC}} \approx 10^{3} \mathrm{pc}^{-3}$. In their proper motion study of the ONC, Jones \& Walker (1988) found the velocity dispersion to be nearly constant at all cluster radii and obtained a one-dimensional velocity dispersion $\sigma_{1 \mathrm{D}}^{\mathrm{JW}}=2.5 \mathrm{~km} \mathrm{~s}^{-1}$. This translates into a three-dimensional velocity dispersion of $\sigma_{3 \mathrm{D}}^{\mathrm{JW}}=\sqrt{3} \sigma_{1 \mathrm{D}}^{\mathrm{JW}}=4.3 \mathrm{~km} \mathrm{~s}^{-1}$. Recently, Furesz et al. (2007) obtained a somewhat higher one-dimensional velocity dispersion of $\sigma_{1 \mathrm{D}}^{\mathrm{F}+}=3.1 \mathrm{~km} \mathrm{~s}^{-1}$ from radial velocity measurements. However, they caution that their velocity distribution of ONC stars has a peak that is too low compared to the expected Gaussian distribution with dispersion $\sigma_{1 \mathrm{D}}^{\mathrm{F}+}$, so we will rely on the result of Jones \& Walker (1988).

Using this value, the virial ratio $Q_{\text {vir }}$ of the ONC becomes

$Q_{\mathrm{vir}}=\frac{R_{\mathrm{hm}}\left(\sigma_{3 \mathrm{D}}^{\mathrm{JW}}\right)^{2}}{2 G M} \approx 1.5$,

where $M=\bar{m} N \approx 2000 M_{\odot}$. This indicates that the ONC seems to be gravitationally unbound $\left(Q_{\text {vir }}>1\right)$. However, the gas mass of the background molecular cloud OMC 1 should be considered. Huff \& Stahler (2006) suggest that the molecular cloud that formed the $\mathrm{ONC}$ contained about $6000 M_{\odot}$. The remaining part of it in the cluster background still has a mass of about $2000 M_{\odot}$ (Hillenbrand et al. 1998).

In the most recent study on circumstellar discs in the Trapezium Cluster, Lada et al. (2000) found a fraction of $80-85 \%$ discs among the stellar population from the $L$-band excess. This is in agreement with an earlier investigation of the complete ONC in which Hillenbrand et al. (1998) report a disc fraction of $50-90 \%$ (though relying only on $I_{C}-K$ colors) and justifies the assumption of a $100 \%$ primordial disc fraction.

The treatment of binaries in our model of the ONC is crucial, since they can have a strong impact on the evolution of cluster dynamics. In the next section we give a summary of the main properties of the ONC binary population that determine our model setup.

\subsection{Binaries in the ONC}

In the ONC the binary rate for solar-type stars is $\sim 50 \%$. From observations alone we have only a very limited knowledge of the distribution of binary periods, eccentricities or mass ratios of the ONC. However, combining observational data and numerical simulations, the initial properties of the primordial binary population in a stellar aggregate have been modelled by Kroupa (1995b) and Kouwenhoven et al. (2007). These can be applied to some degree to the ONC.

The investigation of Kroupa (1995b) is based on the properties of the Taurus-Auriga binary population and constructs the primordial population by inverse dynamical population synthesis (see Kroupa 1995a) and pre-main-sequence eigenevolution. The resulting distributions are approximately the log-normal period distribution $f_{P}(P)$ of Duquennoy \& Mayor (1991),

$f_{P}(P) \propto \exp \left[\frac{\log P-\mu_{P}}{2 \sigma_{P}^{2}}\right], \quad P_{\min } \leq P \leq P_{\max }$,

with mean $\mu_{P} \equiv \overline{\log P}=4.8$ and standard deviation $\sigma_{P} \equiv \sigma_{\log P}=2.3, P$ in days, a thermally relaxed eccentricity distribution $f_{e}(e)$,

$f_{e}(e)=2 e, \quad 0 \leq e<1$,

and a mass ratio distribution $f_{q}(q)$ obtained by random pairing of stars,

$f_{q}(q) \propto q^{\gamma_{q}}$,

where $q=M_{2} / M_{1}, M_{1}$ the primary, $M_{2}$ the secondary mass, and $\gamma_{q}=\alpha, \alpha$ the slope of the mass function of the stellar system.

The log-normal period distribution $f_{P}(P)$ results in an approximately log-normal semi-major axis distribution $f_{a}(a)$, the 
shape of which is slightly dependent on the distribution over binary mass $M$ (Kouwenhoven et al. 2007):

$\overline{\log a}=\frac{2}{3} \overline{\log P}-\frac{1}{3} \log \left(\frac{4 \pi}{2 G M}\right), \quad \sigma_{\log a}=\frac{2}{3} \sigma_{\log P}$

Kouwenhoven et al. (2007) have analyzed the current binary population of Scorpius OB2, under the reasonable assumption that it is still close to its primordial state. Accounting for different observational biases by means of comparison with simulated observations of model associations, they recovered a somewhat different primordial binary population: the semi-major axis distribution of Sco OB2 is most consistent with a flat distribution in logarithmic space, and is equivalent to

$f_{a}(a) \propto a^{\gamma_{a}} \quad a_{\min } \leq a \leq a_{\max }$,

with $a_{\min } \approx 5 R_{\odot}, a_{\max } \approx 5 \times 10^{6} R_{\odot}$, and $\gamma_{a}=-1$, which is also known as Öpiks law (Öpik 1924). The eccentricity distribution could not be well constrained, but the observations are consistent with a thermal distribution, given by Eq. (5). Unlike Kroupa (1995b), Kouwenhoven et al. (2007) find a power law dependence of the mass ratio distribution with $\gamma_{q} \approx-0.4$. This is much flatter than Eq. (6) and favors massive companions for massive stars.

The model of Kouwenhoven et al. (2007) seems more applicable to our case, as observational studies of the ONC favor a flat distribution of the semi-major axes (e.g. Padgett et al. 1997; Reipurth et al. 2007). Theoretical considerations based on threebody encounters give similar results (Valtonen 1997).

A thermal eccentricity distribution is expected from energy equipartition as a result of multiple soft encounters (Heggie 1975 ) and is also found from observations, though only for binaries with separations $a \gtrsim 10-50 \mathrm{AU}$. Very close systems are subject to circularization due to tidal effects occurring during stellar evolution (Duquennoy \& Mayor 1991; Mathieu 1994).

The shape of the observed mass ratio distribution is not well constrained by observations. However, to a good approximation the mass ratio distribution can be described by a power law as given by Eq. (6) over a wide mass range (e.g. Trimble 1990; Malkov \& Zinnecker 2001; Rucinski 2001; Shatsky \& Tokovinin 2002; Valtonen 2004). We thus favor a single mass ratio distribution for primordial binaries given by Eq. (6) with $\gamma_{q}=-0.4$ as derived by Kouwenhoven et al. (2007).

Binaries have strong effects on the overall cluster dynamics mainly through close interactions with single stars or other multiples. In the following we estimate the typical encounters that could generate high-velocity stars.

\subsection{Three-body encounters in the ONC}

As is well known, the non-hierarchical motion of three bodies, known as the three-body problem, has no analytical solution, and the chaotic motion of the members can only be investigated numerically in a statistical manner (see Valtonen \& Mikkola 1991). In her study of triple systems with negative total energy, Anosova (1986) found that about $95 \%$ of three-body systems decay after a close triple approach of the components. In most cases $(\sim 80 \%)$, ejection leads to escape, but can also result in the formation of a hierarchical triple system, with one body in an extended orbit. The lowest mass member has the highest probability of being ejected, about $80 \%$.

In the following we assume that high-velocity stars are typically generated in triple systems with negative total energy. We will justify this assumption later in this section. If the motion has not been significantly perturbed since the encounter, one can draw conclusions about the underlying encounter parameters from the dynamics of the ejected body. In the case of the $\mathrm{ONC}$, it is valid to assume that high-velocity stars with velocities $v \geq 3 \sigma_{3 \mathrm{D}}^{\mathrm{JW}} \approx 13 \mathrm{~km} \mathrm{~s}^{-1}$ are effectively unperturbed before they escape from the cluster (see Appendix C). Then the time to reach the outskirts of the cluster is $t_{\mathrm{esc}} \approx R / v \lesssim 0.2 \mathrm{Myr}$.

For a crude estimate of the compactness of the three-body system (with negative total energy) from which a member is ejected with $v \geq 3 \sigma_{3 \mathrm{D}}^{\mathrm{JW}}$, we evaluate the scaling of the median escape speed from Sterzik \& Durisen (1995),

$\left\langle v_{\mathrm{esc}}\right\rangle \approx \frac{1}{2}\left(\frac{\left|E_{0}\right|}{\left\langle m_{\mathrm{esc}}\right\rangle}\right)$,

where $\left\langle m_{\mathrm{esc}}\right\rangle$ denotes a weighted mean of the escaped particle masses, $\left|E_{0}\right| \propto M_{\mathrm{tot}}^{2} / R$ is the total system energy, and $M_{\mathrm{tot}}$ and $R$ are the total mass and the scale length of the system.

We assume that the encounter occurred in the dense Trapezium Cluster where it is most probable (see Fig. 4 of Pfalzner et al. 2006). Due to mass segregation of the cluster, the mass of the most massive component of the three-body system is likely to be several times the mean stellar mass in the ONC; we adopt $M_{\text {tot }}=4 M_{\odot}$ for the system mass. The mass of the ejected body is assumed to be half the mean stellar mass, $\left\langle m_{\mathrm{esc}}\right\rangle=0.25 M_{\odot}$. With these assumptions we obtain $R \lesssim 100 \mathrm{AU}$ for the scale length of the system. However, the minimum approach which eventually leads to an ejection will be much closer. Since the disc radius of a low-mass star is about $r_{d} \approx 100 \mathrm{AU}$, the ejected component can lose more than $90 \%$ of the disc mass (see Table 3 of Olczak et al. 2006).

Initially we assumed that triple systems that generate highvelocity stars have negative total energy. In Appendix D we show that this is a valid assumption if the ejected high-velocity stars do not exceed velocities of a few tens of $\mathrm{km} \mathrm{s}^{-1}$. In fact, all of the high-velocity stars from numerical simulations and observational data do not show higher velocities. A detailed analysis of our numerical simulations shows that in all cases the encountergenerated high-velocity stars are the lowest mass component ejected from a three-body system with a massive tight binary $\left(M_{\text {bin }} \gtrsim 20 M_{\odot}, a_{\text {bin }} \lesssim 50 \mathrm{AU}\right)$, leaving on a nearly parabolic orbit relative to one of the binary components. We thus conclude that the triple systems which generated the observed low-mass high-velocity stars must have been bound as well.

How many such encounters do we expect in the ONC? For simplicity we assume the three-body interaction to result from a single-binary encounter, where the binary has a semi-major axis $a \lesssim 100 \mathrm{AU}$. As a volume relevant for close encounters we consider the Trapezium Cluster. Referring to Eq. (C.1) the time scale for a three-body encounter at $100 \mathrm{AU}$ is $t_{\mathrm{enc}, 100 \mathrm{AU}} \approx 10 \mathrm{Myr}$. Since the time the star remains in the cluster volume is $t_{\text {esc }} \lesssim 0.2 \mathrm{Myr}$, the probability of the detection of a high-velocity star is $P_{\mathrm{hvs}} \approx t_{\mathrm{esc}} / t_{\mathrm{enc}, 100 \mathrm{AU}} \lesssim 0.02$. Knowing the number of stars located in the Trapezium Cluster, $N_{\mathrm{TC}}$, we expect $N_{\mathrm{hvs}} \approx P_{\mathrm{hvs}} N_{\mathrm{TC}} \lesssim 15$ high-velocity stars in the ONC.

In summary, high-velocity stars with velocities $v \geq 3 \sigma_{3 \mathrm{D}}^{\mathrm{JW}}$ (or velocity components $v_{x, y, z} \geq 3 \sigma_{1 \mathrm{D}}^{\mathrm{JW}}$ ) have most probably experienced exactly one close $(\lesssim 100 \mathrm{AU})$, disruptive encounter in the cluster centre and leave the cluster on a radial trajectory, i.e. with the velocity vector pointing away from the cluster centre, preserving the dynamical information of the encounter. We expect about one dozen such stars in the ONC.

In the following we will compare the observationoal data and theoretical estimates with numerical simulations of a dynamical model of the ONC. 


\section{Numerical simulations}

The basic dynamical model of the ONC used here is described in Olczak et al. (2006), with several extensions discussed in Pfalzner \& Olczak (2007b). We summarize the main properties of the initial stellar distribution: the simulations start in virial equilibrium, $Q=0.5$, with a radial density profile, $\rho \propto r^{-2}$, a central density $\sim 4 \times 10^{5} \mathrm{pc}^{-3}$, and a Maxwellian velocity distribution. Here we include additional effects like a varying background potential and a population of primordial binaries. We have performed 20 runs with different random configurations of positions and velocities from the given distributions to establish a statistically robust database. Unless explicitly declared otherwise the presented results refer to the whole set of runs. The reason to extend our basic model of the ONC is that we want to compare the velocity distribution to the observational data. Both the background potential and primordial binaries have a large impact on the resulting velocity distribution. However, for comparison and analysis of the contribution of the background potential and primordial binaries, we also performed simulations of a single particle model, a single particle model with a background potential, and a cluster with a primordial binary population but without a background potential.

A background potential increases the cluster virial mass and thus results in a higher velocity dispersion (cf. Eq. (3)). In our simulations the background potential is represented by a Plummer sphere,

$\rho(r)=\frac{3 M}{4 \pi a^{3}} \frac{1}{\left(1+\frac{r^{2}}{a^{2}}\right)^{5 / 2}}$,

with mass $M$ and characteristic length scale $a$. The Plummer sphere is set up with $6000 M_{\odot}$ initially and a mass loss rate $\dot{M}=4000 M_{\odot} \mathrm{Myr}^{-1}$. Due to the continuous mass loss, after $1 \mathrm{Myr}, 2000 M_{\odot}$ of gas is left. The exact time-scale and time dependency of the mass loss is not crucial, as long as the gas expulsion time scale is of the order of the dynamical time scale of the system, $t_{\exp } \approx t_{\text {dyn }}$, which appears to be the case for the ONC (cf. Sect. 3). The length scale is set to $a=0.6 \mathrm{pc}$ to match the observed velocity dispersion at a simulation time of $t_{\text {sim }}=1 \mathrm{Myr}$.

The effect of primordial binaries is more complicated. The interactions of binaries and single stars or other binaries have the potential to change the velocity distribution much more than a single star model. In particular, three body encounters between singles and binaries usually lead to the expulsion of the lowest mass member from the small $N$-body system with a high velocity. This mechanism is especially important for the high-velocity fraction of particles of interest in this study (see Sect. 3.2).

We have set up a series of simulations with the models of Kroupa (1995b) and Kouwenhoven et al. (2007) and found that results do not depend on the choice of one particular model. The initial binary frequency was chosen to be $75 \%$. Although the observed binary frequency in the ONC is $\sim 50 \%$, it is necessary to start with a higher binary rate as about one third of the binaries are distroyed within the first 1 Myr due to dynamical evolution.

In terms of global cluster dynamics, the new model with a background potential and primordial binaries provides a much better fit to the ONC data than the previous single star model without background potential. We illustrate this by means of the time-evolution of the three-dimensional velocity dispersion of both models in Fig. 4. In the previous model there was not enough mass confined in the cluster to reproduce the observed velocity dispersion of the $\mathrm{ONC}, \sigma_{3 \mathrm{D}}^{\mathrm{JW}}=4.3 \mathrm{~km} \mathrm{~s}^{-1}$, at $\sim 1 \mathrm{Myr}$. The new model gives a much better result. The continuous, steep falloff after $\sim 0.2 \mathrm{Myr}$ is due to the response of the stellar system

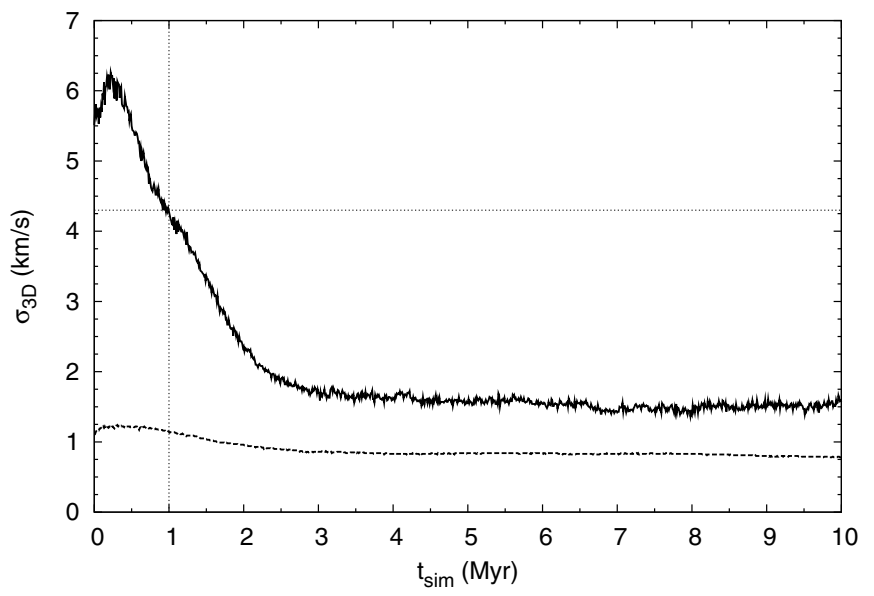

Fig. 4. Three-dimensional velocity dispersion as a function of time for the previous (dashed) and new (solid) numerical models of the ONC. The dotted horizontal line marks the three-dimensional velocity dispersion from Jones \& Walker (1988), $\sigma_{3 \mathrm{D}}^{\mathrm{JW}}=4.3 \mathrm{~km} \mathrm{~s}^{-1}$, the vertical dotted line marks the mean age of the $\mathrm{ONC}, t_{\mathrm{ONC}}=1 \mathrm{Myr}$.

to the gas expulsion. In the following, we will discuss results of numerical simulations based on the new dynamical model of the ONC.

As demonstrated in Olczak et al. (2006), Pfalzner et al. (2006), and Pfalzner \& Olczak (2007a), stellar encounters in dense clusters can lead to significant transport of mass and angular momentum in protoplanetary discs. In the present investigation we have used Eq. (1) from Pfalzner et al. (2006) to keep track of the disc-mass loss of each star due to encounters. Our estimate of the accumulated disc-mass loss is an upper limit because the underlying formula is only valid for co-planar, prograde encounters, which are the most perturbing. A simplified prescription assigns stars into one of two distinct groups: if the relative disc-mass loss exceeds $90 \%$ of the initial disc mass, stars are marked as "discless"; otherwise they are termed "star-disc systems". This approach is justified by the interplay of three effects: (i) A disc-mass loss of this order lowers the density in the disc significantly, in particular in the outer parts; the disc size decreases. (ii) The accompanying angular momentum loss enhances accretion of the extant material onto the star (Pfalzner 2006). This leads temporarily to an increase of the infrared excess but soon fades after a short intense accretion phase (Pfalzner 2008, subm. to A\&A). (iii) The loose distribution of circumstellar matter lowers the shielding of the disc midplane against photoevaporation. The interplay of these effects leads to a fast dispersal of the disc material. From the observational point of view, the corresponding star would show pure photospheric emission on the order of some $10^{3} \mathrm{yr}$ after the encounter.

In Fig. 5 the velocity distribution of cluster stars is shown after $1 \mathrm{Myr}$ of evolution. This is done separately for discless stars and star-disc systems. For comparison with the observational data presented in Fig. 1c, separate velocity distributions of two spacial directions (here $x$ and $y$ ) have been added to mimic the distribution of proper motion data. Unless in wide systems, primaries and secondaries could not have been resolved by Jones \& Walker (1988) who worked with seeing-limited images. So the presence of binaries in the numerical simulations requires a special treatment of velocities. Accounting for unequal mass components and nebulosity, we adopt a minimum separation of $1000 \mathrm{AU}$ (corresponding to 2.5"; see Mayne \& Naylor 2008, and references therein) for the visual resolution 


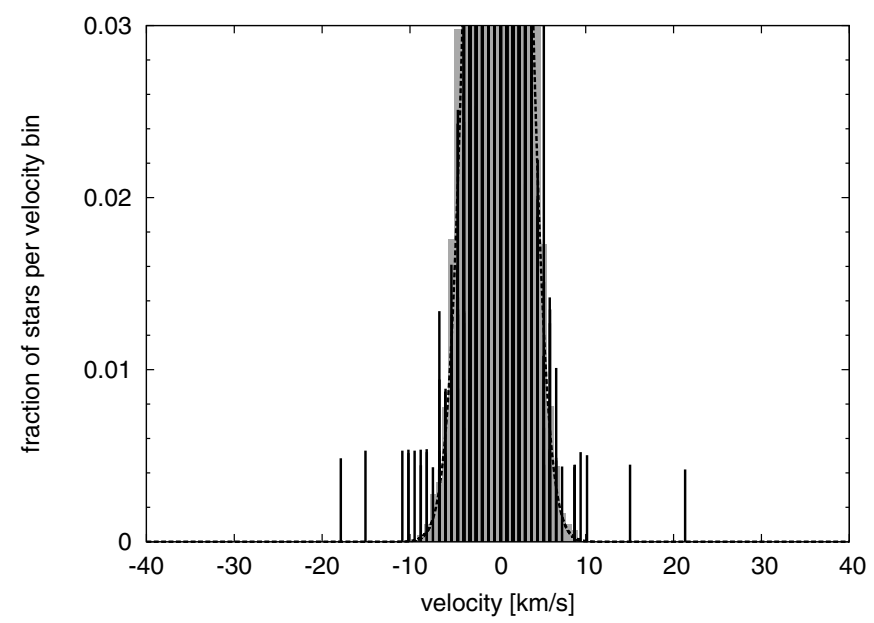

Fig. 5. Velocity distribution of cluster stars from simulations. The construction of the distribution is described in the text. The bin width is $0.7 \mathrm{~km} \mathrm{~s}^{-1}$. The stars have been divided into two groups according to their extant disc mass (see text): boxes represent star-disc systems, impulses represent discless stars. For comparison a Gaussian with dispersion $\sigma=\sigma_{1 \mathrm{D}}^{\mathrm{JW}}=2.5 \mathrm{~km} \mathrm{~s}^{-1}$ is superposed (dashed line).

of a binary system. For closer systems, only the primary component is taken into account and the center-of-mass velocity of the system is assigned. For wider systems, both components are treated as single stars. This prescription is simple and rough but appropriate to avoid the inclusion of large velocity components in tight binaries.

The velocity distribution shows common features with Fig. 1c, which was obtained from observational data: the bulk of the stars forms a relaxed system which manifests in the approximate Gaussian velocity distribution with a characteristic velocity dispersion $\sigma=\sigma_{1 \mathrm{D}}^{\mathrm{JW}}$. Moreover, a small fraction of stars exists with much higher velocities $v_{x, y} \geq 3 \sigma_{1 \mathrm{D}}^{\mathrm{JW}}$, the previously described "high-velocity stars".

The velocity distributions of stars that have lost their disc due to close encounters and those that have retained their disc differ. The discless population consists of a larger fraction of high-velocity stars, while the width of the Gaussian part is similar. This feature is in agreement with expectations: high-velocity stars are usually the lowest mass members of temporary fewbody systems which are expelled after a close encounter (see Sect. 3.2). The close passage and large mass of the perturber results in a significant removal of disc material (Pfalzner et al. 2006).

In analogy to Fig. 3, positions and velocities of high-velocity stars from the numerical simulations are displayed in Fig. 6. Here the great advantage of numerical simulations becomes apparent: several runs of the same model can improve statistics far enough to produce prominent features where only weak signatures in observational data are found. Our dynamical model of the ONC reproduces the observed features: as expected, most stars are concentrated in the inner tenth parsecs around the cluster centre, while several stars are located in the outer cluster parts, moving in radial directions from the cluster centre. However, we find the same two unexpected features as in the observations, namely that (i) the region between the cluster centre and the outer cluster parts is underpopulated by high-velocity stars; and (ii) the velocity vectors of a fraction of stars in the outer cluster parts do not point away from the cluster centre. We will refer to high-velocity stars that leave the cluster on a track in



Fig. 6. Positions and velocities of high-velocity star-disc systems (open circles) and discless stars (filled circles) from cluster simulations. The frame is centered on the cluster centre. The lines indicate the distance (in pc) a star would have moved in a period of $2 \times 10^{4} \mathrm{yr}$.

radial direction from the cluster centre, i.e. with the velocity vector pointing away from the cluster core with radius $R_{\text {in }} \approx 0.1 \mathrm{pc}$, as "radial escapers", while high-velocity stars that do not match this condition will be called "orbital escapers". In the following we explain the choice of the terminology and discuss the two classes of high-velocity stars.

In contrast to observations we can trace the history of these stars in the simulation; this gives us the opportunity to investigate the reason for this strange configuration. As we will see, (i) is a consequence of (ii), thus we will discuss that first. In Fig. 7 two exemplary tracks of stars in the outer cluster parts are displayed, those of a radial (Fig. 7b) and an orbital escaper (Fig. 7a). It is evident that the phase spaces of the two stars are completely different. The radial escaper was originally located close to the cluster centre, experienced several encounters in multiple passages of the cluster centre and was finally expelled in a close three body encounter. After the ejection, it is moving on a track in a radial direction from the cluster centre, i.e. with the velocity vector pointing away from the cluster core. The orbital escaper stems from the outer cluster parts, passed on a non-closed orbit at a minimum distance of some tenths of a parsec around the cluster centre (without significant encounters) and was accelerated sufficiently by the central mass to leave the cluster on a hyperbolic orbit. Most of the time its velocity vector is not pointing away from the cluster core. This is most evident at large distances from the cluster centre. Only at the two short periods of cluster centre passage, i.e. when the radius vector is approximately normal to the velocity vector, the determination of the direction of motion is eventually not sufficient to discriminate between a radial and orbital escaper. The fact that the orbital escaper is leaving the cluster although it was initially energetically (but only weakly) bound to the cluster is due to a varying cluster potential on a 
a)

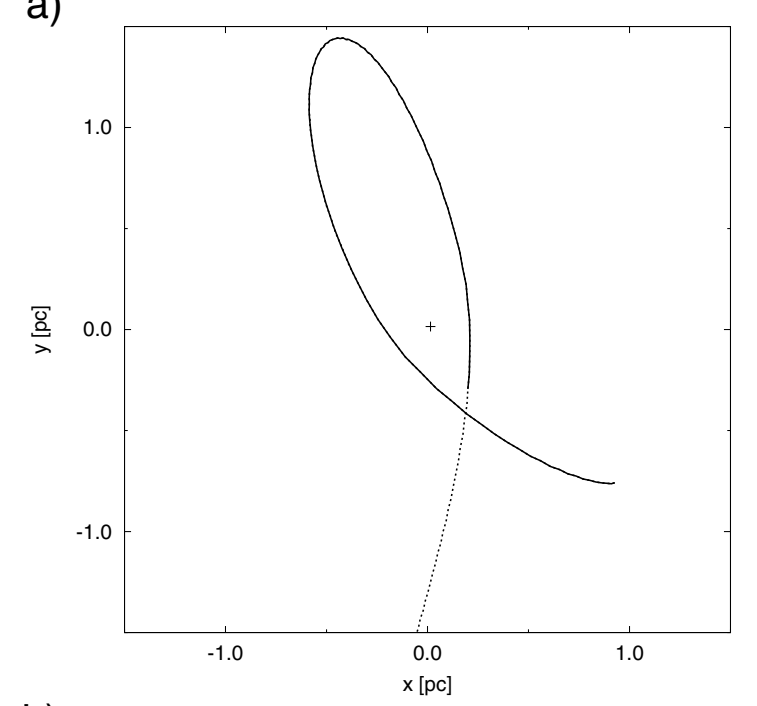

b)

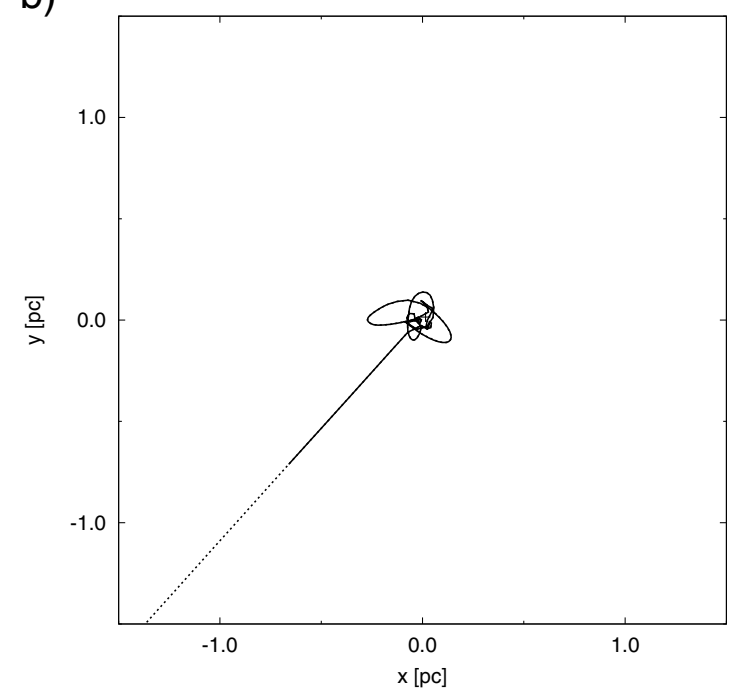

Fig. 7. Tracks of two high-velocity stars from simulations. The cross marks the cluster centre. The solid line marks the trajectory up to $1 \mathrm{Myr}$, the dotted line represents later times. a) Star on a wide orbit around the cluster centre, escaping from the cluster after a close passage of the cluster centre. b) Star escaping after multiple passages of the cluster centre and a final close encounter.

time scale shorter than its revolution around the cluster centre ${ }^{2}$. The main sources of the potential variation are mass segregation and the evaporation of (preferentially low-mass) cluster stars. Consequently the orbital escaper is accelerated more strongly after the second passage of the cluster centre and is less decelerated in the outer parts due to the lower total cluster mass and more extended cluster potential.

The existence of two different classes of tracks explains why the region between the cluster centre and the outer cluster parts is devoid of high-velocity stars. Radial escapers leave the cluster on a very short time scale, while orbital escapers reside for much longer in the vicinity of the cluster centre due to their wide nonclosed orbits. Hence the disjoint spacial groupings of stars after

\footnotetext{
2 The time scale of the potential variation is related to the crossing time of the cluster, $t_{\text {cross }} \approx 0.5 \mathrm{Myr}$, while the time scale of revolution is approximately the cluster age, $t_{\mathrm{ONC}} \approx 1 \mathrm{Myr}$, which is about twice as large.
}

1 Myr of cluster evolution are the consequence of disjoint sets of initial phase space volumes.

We find from our simulations that the distinct dynamics of the two exemplary high-velocity stars characterize in general the dynamics of radial and orbital escapers: they belong to dynamically distinct groups. Moreover, the two groups as well can be separated due to their disc properties: a large fraction of radial escapers is discless, while most orbital escapers are star-disc systems. This morphological distinction is a consequence of the dynamical bisection. The morphological bisection translates observationally into a photometrical bisection, i.e. the stars would be devided into two groups according to the presence of excess emission. Such a trend, though only weak, is also present in the observational data.

In Fig. 3b, the three isolated stars with excess emission are identified as orbital escapers. The situation is more diffcult for the two stars with excess emission immediatly below the cluster centre. As mentioned above, stars close to the cluster centre cannot be uniquely identified as radial or orbital escapers from the direction of motion alone. Moreover, we have only two-dimensional spacial and velocity information, so the true distances to the cluster centre and velocities are not known. If the projected distance and true distance to the cluster centre differ only slightly for both stars, then we would classify the more distant $(\sim 0.25 \mathrm{pc})$ as a probable orbital escaper. The reason is that due to its proximity to the cluster centre and relatively low velocity this star will be accelerated and deflected by the central cluster mass and pass on a curved trajectory. When passing the cluster outskirts, its direction of motion would not point away from the cluster core and thus it would be identified as an orbital escaper according to our classification scheme. Of course, we cannot exclude the possibility that this star was ejected in a three-body encounter, although it could not be classified as a radial escaper due to its predicted trajectory. For the other star a classification as a radial escaper seems more appropriate. If, on the contrary, the true distance is much larger than the projected distance for both stars, they would be classified as orbital escapers. However, we can only speculate about the dynamical origin of the two stars. The special case of the two close-by stars with excess emission in the lower left will be discussed below. The only star without excess emission (JW 45) seems to have been expelled very close $(<0.13 \mathrm{pc})$ to the cluster centre and is thus classified as a radial escaper. If we thus interpret, conversely to the previous arguments, the lack of excess emission as an indicator of the absence of a disc, then this star provides evidence for encounter-triggered disc destruction. The signature of disc material of the other stars is - as far as a classification is possible in accordance with our numerical results and thus supports this view.

The two close-by stars at approximately $(-1.5 \mathrm{pc},-1.0 \mathrm{pc})$ in Fig. 3, JW 3 and JW 4, seem to form a binary. Though their separation of about $10^{4} \mathrm{AU}$ is large, the remarkably similar proper motions and radial velocity $\left(v_{r}^{\mathrm{JW} 3}=29.1 \mathrm{~km} \mathrm{~s}^{-1}\right.$, $v_{r}^{\mathrm{JW}}{ }^{4}=31.6 \mathrm{~km} \mathrm{~s}^{-1}$ : Stassun et al. 1999), age, and infrared excess strongly support the assumption of a physical pair - at least in the past. If this pair was expelled as a binary from a fourbody encounter, than this must have occurred less than $0.1 \mathrm{Myr}$ ago (accounting for the actual distance from the cluster centre, the velocity and the deceleration by the interior cluster mass). The difference in proper motion of the two stars corresponds to a distance of $\sim(4 \pm 2) \times 10^{4} \mathrm{AU}$, in good agreement with the observed projected separation. Due to the direction of motion, the two stars are classified as radial escapers. The expulsion of binaries from close four-body encounters in our simulations, though 


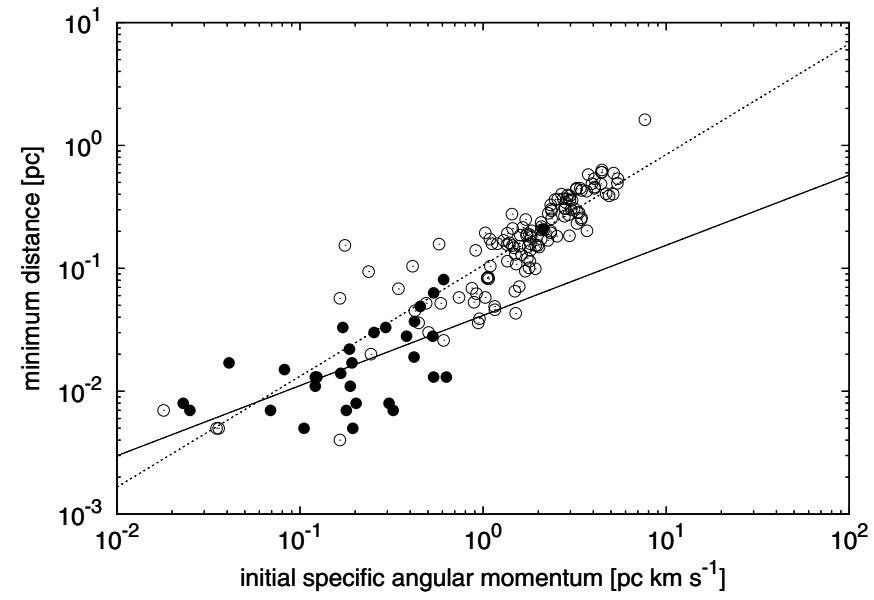

Fig. 8. Minimum distance of the high-velocity stars to the cluster centre, $d_{\min }$, as a function of the initial specific angular momentum, $l_{0}$ (relative to the cluster centre). Star-disc systems are marked by open, discless stars by filled circles. Linear best-fits of both populations, star-disc systems (dotted line) and discless stars (solid line), have been included (quantitative results are discussed in the text).

a rare event ( 9 events from our 20 runs), usually does not lead to a significant disc-mass loss of the individual stars. This again is in good agreement with the observational data. Thus the excess emission of the binary radial escaper does not contradict the correlation of our dynamical and the photometric classification, which in the case of single stars shows that in most cases radial escapers are discless and orbital escapers are found preferentially among star-disc systems.

The general difference of the orbits of discless stars and stardisc systems is represented in Fig. 8. The minimum distance of star-disc systems to the cluster centre $d_{\text {min }}$ is clearly a linear function of the initial specific angular momentum $l_{0}$ (relative to the cluster centre). From a linear least squares fit we find a slope $\alpha$ with a small standard error $\Delta \alpha, \alpha=(0.902 \pm 0.044) \mathrm{s} \mathrm{km}^{-1}$ $(\Delta \alpha / \alpha=0.048)$. This means that $l_{0}$ is conserved - a consequence of the wide orbit around the cluster centre without strong, abrupt perturbations from single stars. On the contrary, discless stars show a much wider, not clearly correlated distribution of $d_{\min }$ due to angular momentum exchange in close encounters in the cluster centre. Here the slope $\beta$ of the linear best-fit has a large standard error $\Delta \beta, \beta=(0.571 \pm 0.127) \mathrm{s} \mathrm{km}^{-1}$ $(\Delta \beta / \beta=0.222)$. Those star-disc systems with lower $l_{0}$ and $d_{\min }$, populating the discless regime, are components of binaries.

The distinct dynamics are even more evident from Fig. 9: here we show the ratio of the actual (at $1 \mathrm{Myr}$ ) and initial specific angular momentum $l_{\text {now }} / l_{0}$ as a function of the initial specific angular momentum $l_{0}$. Star-disc systems are concentrated nearly symmetrically around $l_{\text {now }} / l_{0}=1$, while for most discless stars $l_{\text {now }} / l_{0}>1$, and even up to several tens. The increase of angular momentum of the discless stars in relation to the cluster centre can be explained as follows: Single stars gain a large amount of angular momentum in a close triple encounter (Valtonen et al. 2005), and leave on straight radial tracks after breakup. Since these encounters occur preferentially close to the cluster centre, on average the angular momentum relative to the cluster centre is also highly increased. The raise of $l_{\text {now }} / l_{0}$ with lower $l_{0}$ is given by the fact that close encounters are more probable for stars with lower $l_{0}$, which in turn lead to a higher gain in angular momentum.

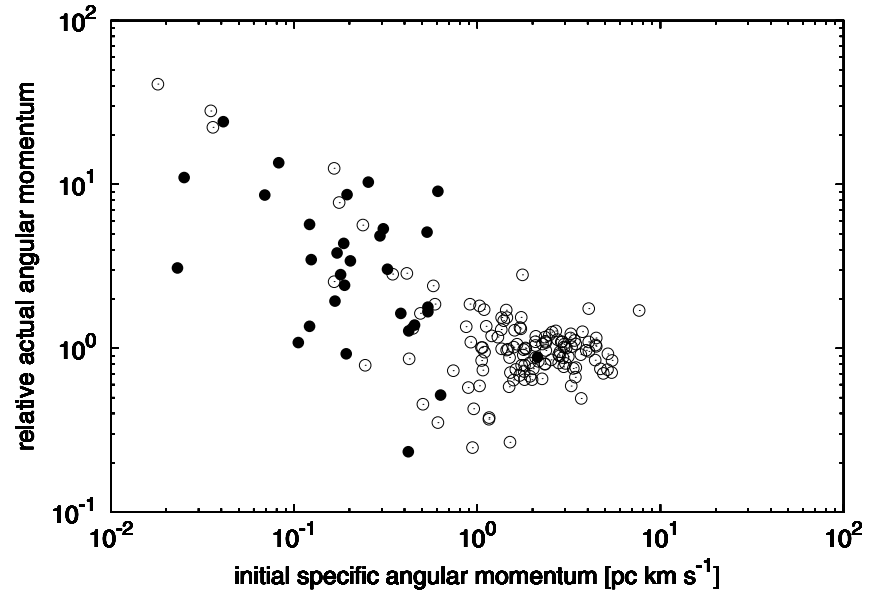

Fig. 9. Relative actual angular momentum of the high-velocity stars, $l_{\text {now }} / l_{0}$, as a function of the initial specific angular momentum $l_{0}$ (relative to the cluster centre). Star-disc systems are marked by open, discless stars by filled circles.

The additional components in our numerical model of the ONC - a background potential and primordial binaries - have different effects on the sample of high-velocity stars. By comparing with results of our simulations without either one or both additional components, we find that (i) the inclusion of primordial binaries has the effect of increasing the number of discless stars (due to a larger number of encounters) and to increase the maximum velocity of the high-velocity stars (due to a higher probability of closer encounters); (ii) a background potential reduces the number of discless stars and reduces the maximum velocity of the high-velocity stars, because the higher velocity dispersion of the stars reduces the probability of close encounters. The combination of both as in the present model does not cancel out the positive effect of the binaries and thus results in a higher number of discless stars and a higher maximum velocity of the high-velocity stars than would result from a single star model.

\section{Summary and discussion}

Combining observational data and numerical simulations, we have shown that even after 1 Myr of dynamical evolution a cluster of the size and density of the ONC is still dynamically active. Young stars, preferentially of low-mass, are expelled in close $\mathrm{N}$-body encounters, losing a large fraction of their circumstellar matter.

The encounters are in most cases interactions with massive stars in the cluster centre. This finding highlights the significant effect that encounters in (massive) young stellar clusters can have on the evolution of protoplanetary discs. This is even more evident if one addresses not only the effect on the mass, but the even stronger effect on the angular momentum of the disc as shown in previous investigations (Pfalzner 2006; Pfalzner \& Olczak 2007a).

Using the observational data from Jones \& Walker (1988) we show (Fig. 1c) that in the ONC there is a small population of stars with proper motions $\mu_{x, y}$ larger than three times the onedimensional velocity dispersion of the cluster, $\mu_{x, y} \gtrsim 7.5 \mathrm{~km} \mathrm{~s}^{-1}$.

From numerical simulations we find that the so-called "highvelocity stars" form two dynamically disjoint groups. One group is composed of stars expelled in a close encounter, moving on 
radial tracks directly outward from the cluster centre, termed "radial escapers". The other contains unperturbed stars running on wide, non-closed orbits around the cluster centre (on average with lower velocities), termed "orbital escapers". The dynamical state of the stars has its origin in their initial phase space location: radial escapers are initially located close to the cluster centre $(r \lesssim 0.3 \mathrm{pc})$ and characterized by a low angular momentum (relative to the cluster centre). In contrast, orbital escapers were formed in the outer cluster with large angular momentum relative to the cluster centre.

The different dynamics of the high-velocity stars implies a signature in their circumstellar characteristics: stars being subject to close encounters are expected to lose their disc material faster and to a higher degree (Pfalzner et al. 2006). Using our prescription of disc-mass loss in stellar encounters, indeed we find a clear correlation from numerical simulations: radial escapers, initially located close to the cluster centre and later expelled in a close encounter, lose more than $90 \%$ of their disc material in $1 \mathrm{Myr}$ of dynamical evolution, while orbital escapers, initially distant stars, moving on wide orbits around the cluster centre, do not experience strong perturbations and keep most of their disc material.

We compare our numerical results with infrared observations from Hillenbrand (1997), Muench et al. (2002), and Lada et al. (2004), tracing the (inner) circumstellar material. Though strict conclusions are not possible due to the small observational sample and some stars that cannot be classified according to our scheme, we observe the same trend: stars that have been classified as orbital escapers do show near-infrared excess emission, indicative of circumstellar matter, while those classified as radial escapers show pure photospheric colors, lacking evidence of (inner) circumstellar discs.

This view is strongly supported by the work of Tan (2004) on the dynamics of $\theta^{1} \mathrm{C}$ Ori. The most massive star in the ONC, $\theta^{1} \mathrm{C}$ Ori, has a proper motion several times greater than the dispersion of bright ONC stars and much larger than the velocity expected if it were in equipartition with the other cluster stars (van Altena et al. 1988). Tan (2004) showed that the direction of $\theta^{1} \mathrm{C}$ Ori's motion is consistent with being exactly opposite to that of a B-type star embedded in the background molecular cloud, the so-called BN object, and concludes that it is most probably a runaway star originating from the Trapezium ejected about 4000 years ago after a close encounter with $\theta^{1} \mathrm{C}$ Ori. This supports the idea that stellar encounters may be likely events in such dense regions as the ONC, or at least in their cores. The evidence that the most massive stellar object was involved in a close encounter with another massive star is in best accordance with the work of Moeckel \& Bally (2007a) and Pfalzner \& Olczak (2007b).

Considering the dynamical age of the ONC of several crossing times, encounters must have had much stronger impacts on a stellar disc at an early age of the cluster, when densities were much higher but massive stars already had formed. Indeed, our simulations confirm this expectation, giving rise to an era of strong and frequent interactions among star-disc systems at the onset of massive star formation.

We expect that in even denser clusters such as the Arches cluster, high-velocity stars should be even more frequent than in the ONC. In such systems the current spatial and velocity distribution of the high-velocity stars should give strong indications of how the cluster developed in former times.

Acknowledgements. We thank the anonymous referee for careful reading and very useful comments and suggestions which improved this work. We are grateful to B. Jones for providing us the proper motion data of the ONC. We also thank R. Spurzem for providing the NBODY $6++$ code for the cluster simulations. Simulations were partly performed at the John von Neumann Institute for Computing, Research Centre Jülich, Project HKU14. This research has made use of the SIMBAD database and the VizieR catalogue access tool, operated at CDS, Strasbourg, France.

\section{Appendix A: Observability of tidal tails due to star-disc encounters in the ONC}

The relevant physical quantity that determines the prominence of tidal tails due to an encounter is the change of angular momentum in the disc. According to Pfalzner \& Olczak (2007a), we assume that a fractional angular momentum loss (AML) of at least $10 \%$ is required to form observationally detectable tidal tails (see also Figs. 9 and 10 of Pfalzner \& Olczak 2007a).

To have an estimate of the rate of encounters in the ONC in which the AML is at least $10 \%$, we consider an encounter of a star with mass $m=0.5 M_{\odot}$, which corresponds to the mean stellar mass in the ONC (see Sect. 3). A star of that mass is thought to be surrounded by a plotoplanetary disc of typical size $r_{\mathrm{d}}=100 \mathrm{AU}$. To be on the safe side, we want to estimate the upper limit of the encounter rate and further assume the encounter partner to be the highest mass star of the ONC, $M=50 M_{\odot}$. Referring to Table 1 of Pfalzner \& Olczak (2007a), the specified minimum AML requires an encounter at a relative periastron $r_{\mathrm{p}} / r_{\mathrm{d}} \approx 10$, or a periastron $r_{\mathrm{p}} \approx 1000 \mathrm{AU}$. Moreover, we assume that the encounter occurred in the Trapezium Cluster (TC), the dense central part of the ONC, where it is most probable (see Fig. 4 of Pfalzner et al. 2006). The number of stars, the density and the velocity dispersion of the TC are $N_{\mathrm{TC}} \approx 750, n_{\mathrm{TC}} \approx 10^{3} \mathrm{pc}^{-3}$, and $\sigma_{1 \mathrm{D}}^{\mathrm{JW}}=2.5 \mathrm{~km} \mathrm{~s}^{-1}$ (see Sect. 3).

The time scale for encounters of the assumed type is given by Eq. (C.1); substituting $n=n_{\mathrm{TC}}, \sigma=\sigma_{1 \mathrm{D}}^{\mathrm{JW}}, r_{*}=r_{\mathrm{p}}$, and $m_{*}=M$, we arrive at $t_{\mathrm{enc}} \approx 2 \times 10^{5} \mathrm{yr}$. With a dissipation time scale of the tidal tails of $t_{\text {diss }} \lesssim 1000 \mathrm{yr}$, the probability of a detection is roughly $P_{\mathrm{obs}} \lesssim t_{\mathrm{diss}} / t_{\mathrm{enc}} \approx 5 \times 10^{-3}$. With the number of stars in the TC, $N_{\mathrm{TC}}$, we expect at most $N_{\mathrm{obs}} \lesssim P_{\mathrm{obs}} N_{\mathrm{TC}} \approx 4$ stars to be accompanied by tidal tails that could be observed at the current time.

\section{Appendix B: Estimate of the mean uncertainty of stellar ages}

Since individual errors of the derived stellar ages are not provided by Hillenbrand (1997), we estimate the mean error from the quoted observational and theoretical uncertainties and the constructed HR diagram. The uncertainties of the derived luminosities, $\log \left(L_{*} / L_{\odot}\right) \lesssim 0.2$, translate into an age uncertainty of $\sim 0.2-0.4$ dex for stars with masses $1.0-0.1 M_{\odot}$. Uncertainties of the derived effective temperatures, $\log T_{\text {eff }} \lesssim 0.02$, translate into an age uncertainty of typically $\sim 0.3 \mathrm{dex}$, but can be as large as $\sim 1$ dex for stars with mass $\lesssim 0.1 M_{\odot}$. Further uncertainties of the derived ages are introduced due to differences between different pre-main sequence evolutionary tracks, which can be as large as 0.6 dex. Assuming a typical uncertainty of 0.3 dex due to uncertainties from luminosity, effective temperature and evolutionary tracks we estimate a mean uncertainty of stellar ages of $\sim 0.5 \mathrm{dex}$.

\section{Appendix C: Minimum velocity for unperturbed escape of stars in the ONC}

High-velocity stars that have been generated due to a close triple encounter in the cluster centre are expected to leave the cluster without significant perturbation. This is due to the fact that 
(a) the fractional change of the velocity $v$ of a high-velocity star is less than $10 \%$ unless the impact parameter is not lower than 100 AU (Binney \& Tremaine 1987, Eq. (4-8)), and (b) only a small fraction of stars experiences more than one encounter closer than $100 \mathrm{AU}$ in one crossing time of the ONC (Scally \& Clarke 2001; Olczak et al. 2006). Alternatively, one can evaluate the collision time scale of the escaper and a binary with semimajor axis $a \approx 100$ AU in the ONC (Binney \& Tremaine 1987, Eq. (8-123)),

$t_{\text {coll }}=\left[16 \sqrt{\pi} n \sigma r_{*}^{2}\left(1+\frac{G m_{*}}{2 \sigma^{2} r_{*}}\right)\right]^{-1} \approx 10 \mathrm{Myr}$,

where the gravitational focusing of a $10 M_{\odot}$ binary in the dense Trapezium Cluster has been considered in order to obtain a robust lower limit on $t_{\text {coll }}$. We have used $r_{*}=a, n=n_{\mathrm{TC}} \approx 10^{3} \mathrm{pc}^{-3}$ and $\sigma=\sigma_{3 \mathrm{D}}^{\mathrm{JW}}=\sqrt{3} \sigma_{1 \mathrm{D}}^{\mathrm{JW}}=4.3 \mathrm{~km} \mathrm{~s}^{-1}$ (see Sect. 3). Since the collision time scale is much longer than the time to reach the cluster outskirts, $t_{\text {coll }} \gtrsim 10 \mathrm{Myr} \gg 0.2 \mathrm{Myr} \gtrsim t_{\text {esc }}$, it is valid to assume that stars with velocities $v \geq 3 \sigma_{3 \mathrm{D}}^{\mathrm{JW}}$ are effectively unperturbed before they escape from the cluster.

\section{Appendix D: Maximum velocity of a star ejected from a bound triple system}

We analyze a triple system after the ejection event forming a configuration of a binary and an escaping body (cf. Valtonen et al. 2005). The total energy of the system is then

$E_{0}=\frac{1}{2} m \dot{r}_{\mathrm{s}}^{2}-G \frac{m_{\mathrm{B}} m_{\mathrm{s}}}{r_{\mathrm{s}}}+\frac{1}{2} M \dot{r}^{2}-G \frac{m_{\mathrm{a}} m_{\mathrm{b}}}{r}$,

where $r_{\mathrm{s}}$ is the separation of the third body relative to the barycentre of the binary, $r$ the separation of the binary components, $m_{\mathrm{s}}, m_{\mathrm{a}}$ and $m_{\mathrm{b}}$ the masses of the escaper and the binary components, $m_{\mathrm{B}}=m_{\mathrm{a}}+m_{\mathrm{b}}$ the binary mass, and $M=m_{\mathrm{a}} m_{\mathrm{b}} / m_{\mathrm{B}}$ and $m=m_{\mathrm{B}} m_{\mathrm{S}} /\left(m_{\mathrm{B}}+m_{\mathrm{s}}\right)$ the reduced masses.

When observing, the detection of the escaper will usually occur when the distance to the binary components greatly exceeds the size of the binary system, $r_{\mathrm{s}} \gg r$. Thus we can neglect the second term in Eq. (D.1). Moreover, in most cases the ejected body will be the lowest mass component of the triple system, $m_{\mathrm{S}} \ll m_{\mathrm{B}}$ (see Sect. 3.2), hence Eq. (D.1) reduces to

$E_{0}=\frac{1}{2} m_{\mathrm{s}} \dot{r}_{\mathrm{s}}^{2}+\frac{1}{2} M \dot{r}^{2}-G \frac{m_{\mathrm{a}} m_{\mathrm{b}}}{r}=\frac{1}{2} m_{\mathrm{s}} \dot{r}_{\mathrm{s}}^{2}-G \frac{m_{\mathrm{a}} m_{\mathrm{b}}}{2 a}$,

where the last term denotes the total binary energy in the general case of an elliptical orbit with semi-major axis $a$.

If we use the same parameters for the triple system as in Sect. 3.2 and assume a nearly equal-mass binary, $m_{\mathrm{s}}=0.25 M_{\odot}$, $2 a=100 \mathrm{AU}, m_{\mathrm{a}} m_{\mathrm{b}} \approx 4 M_{\odot}$, we find from Eq. (D.2) the maximum velocity of the escaper from a triple with negative total energy, $v_{\mathrm{s}}=\dot{r}_{\mathrm{s}} \lesssim 18 \mathrm{~km} \mathrm{~s}^{-1}$. In fact, our simulations show that the binary that generates a high-velocity star never is less massive then $10 M_{\odot}$ and usually exceeds $20 M_{\odot}$, and the mass ratio is never below $1 / 6$ and usually about $1 / 3$. Using these parameters, we find $v_{\mathrm{s}} \lesssim 30-80 \mathrm{~km} \mathrm{~s}^{-1}$.

\section{References}

Adams, F. C., Hollenbach, D., Laughlin, G., \& Gorti, U. 2004, ApJ, 611, 360 Adams, F. C., Proszkow, E. M., Fatuzzo, M., \& Myers, P. C. 2006, ApJ, 641, 504 Anosova, J. P. 1986, Ap\&SS, 124, 217

Balog, Z., Muzerolle, J., Rieke, G. H., et al. 2007, ApJ, 660, 1532
Beust, H., Reche, R., \& Augereau, J.-C. 2005, in Protostars and Planets V, 8092 Binney, J., \& Tremaine, S. 1987, Galactic dynamics (Princeton, NJ, Princeton University Press, 1987), 747

Cabrit, S., Pety, J., Pesenti, N., \& Dougados, C. 2006, A\&A, 452, 897

Clarke, C. J., \& Pringle, J. E. 1991, MNRAS, 249, 584

Currie, T., Kenyon, S. J., Balog, Z., et al. 2008, ApJ, 672, 558

Duquennoy, A., \& Mayor, M. 1991, A\&A, 248, 485

Furesz, G., Hartmann, L. W., Megeath, S. T., Szentgyorgyi, A. H., \& Hamden, E. T. 2007, 711, ArXiv e-prints

Haisch, K. E., Lada, E. A., \& Lada, C. J. 2000, AJ, 120, 1396

Haisch, Jr., K. E., Lada, E. A., \& Lada, C. J. 2001, ApJ, 553, L153

Haisch, Jr., K. E., Jayawardhana, R., \& Alves, J. 2005, ApJ, 627, L57

Heggie, D. C. 1975 , MNRAS, 173, 729

Heller, C. H. 1995, ApJ, 455, 252

Hillenbrand, L. A. 1997, AJ, 113, 1733

Hillenbrand, L. A. 2002, [arXiv:astro-ph/0210520]

Hillenbrand, L. A. 2005, [arXiv:astro-ph/0511083]

Hillenbrand, L. A., \& Carpenter, J. M. 2000, ApJ, 540, 236

Hillenbrand, L. A., \& Hartmann, L. W. 1998, ApJ, 492, 540

Hillenbrand, L. A., Strom, S. E., Calvet, N., et al. 1998, AJ, 116, 1816

Huff, E. M., \& Stahler, S. W. 2006, ApJ, 644, 355

Jeffries, R. D. 2007, MNRAS, 376, 1109

Jones, B. F., \& Walker, M. F. 1988, AJ, 95, 1755

Kouwenhoven, M. B. N., Brown, A. G. A., Portegies Zwart, S. F., \& Kaper, L. 2007, A\&A, 474, 77

Kroupa, P. 1995a, MNRAS, 277, 1491

Kroupa, P. 1995b, MNRAS, 277, 1507

Kroupa, P. 2002, Science, 295, 82

Lada, C. J., \& Lada, E. A. 2003, ARA\&A, 41, 57

Lada, C. J., Muench, A. A., Haisch, Jr., K. E., et al. 2000, AJ, 120, 3162

Lada, C. J., Muench, A. A., Lada, E. A., \& Alves, J. F. 2004, AJ, 128, 1254

Lin, S.-Y., Ohashi, N., Lim, J., et al. 2006, ApJ, 645, 1297

Luhman, K. L., Rieke, G. H., Young, E. T., et al. 2000, ApJ, 540, 1016

Malkov, O., \& Zinnecker, H. 2001, MNRAS, 321, 149

Mathieu, R. D. 1994, ARA\&A, 32, 465

Mayne, N. J., \& Naylor, T. 2008, ArXiv e-prints, 801

McCaughrean, M., Zinnecker, H., Andersen, M., Meeus, G., \& Lodieu, N. 2002, The Messenger, 109, 28

Menten, K. M., Reid, M. J., Forbrich, J., \& Brunthaler, A. 2007, A\&A, 474, 515

Meyer, M. R., Calvet, N., \& Hillenbrand, L. A. 1997, AJ, 114, 288

Moeckel, N., \& Bally, J. 2006, ApJ, 653, 437

Moeckel, N., \& Bally, J. 2007a, ApJ, 661, L183

Moeckel, N., \& Bally, J. 2007b, ApJ, 656, 275

Muench, A. A., Lada, E. A., Lada, C. J., \& Alves, J. 2002, ApJ, 573, 366

Olczak, C., Pfalzner, S., \& Spurzem, R. 2006, ApJ, 642, 1140

Öpik, E. 1924, Tartu Obs. Publ., 25, 6

Padgett, D. L., Strom, S. E., \& Ghez, A. 1997, ApJ, 477, 705

Palla, F., \& Stahler, S. W. 2000, ApJ, 540, 255

Pfalzner, S. 2006, ApJ, 652, L129

Pfalzner, S., \& Olczak, C. 2007a, A\&A, 462, 193

Pfalzner, S., \& Olczak, C. 2007b, A\&A, 475, 875

Pfalzner, S., Olczak, C., \& Eckart, A. 2006, A\&A, 454, 811

Reipurth, B., Guimarães, M. M., Connelley, M. S., \& Bally, J. 2007, AJ, 134, 2272

Rucinski, S. M. 2001, AJ, 122, 1007

Scally, A., \& Clarke, C. 2001, MNRAS, 325, 449

Scally, A., Clarke, C., \& McCaughrean, M. J. 2005, MNRAS, 358, 742

Shatsky, N., \& Tokovinin, A. 2002, A\&A, 382, 92

Sicilia-Aguilar, A., Hartmann, L. W., Szentgyorgyi, A. H., et al. 2005, AJ, 129, 363

Sicilia-Aguilar, A., Hartmann, L., Calvet, N., et al. 2006, ApJ, 638, 897

Slesnick, C. L., Hillenbrand, L. A., \& Carpenter, J. M. 2004, ApJ, 610, 1045

Stassun, K. G., Mathieu, R. D., Mazeh, T., \& Vrba, F. J. 1999, AJ, 117, 2941

Sterzik, M. F., \& Durisen, R. H. 1995, A\&A, 304, L9

Tan, J. C. 2004, ApJ, 607, L47

Trimble, V. 1990, MNRAS, 242, 79

Valtonen, M. J. 1997, ApJ, 485, 785

Valtonen, M. 2004, Rev. Mex. Astron. Astro. Conf. Ser., ed. C. Allen \& C. Scarfe, 21, 147

Valtonen, M., \& Mikkola, S. 1991, ARA\&A, 29, 9

Valtonen, M., Mylläri, A., Orlov, V., \& Rubinov, A. 2005, MNRAS, 364, 91

van Altena, W. F., Lee, J. T., Lee, J.-F., Lu, P. K., \& Upgren, A. R. 1988, AJ, 95, 1744

Wilson, T. L., Filges, L., Codella, C., Reich, W., \& Reich, P. 1997, A\&A, 327, 1177

Zinnecker, H., \& Yorke, H. W. 2007, ARA\&A, 45, 481 